In this attempt to find a method by which the existence of two antigenic varieties within the group of Bacillus botulinus could be established without the recurrence to the toxin-antitoxin test we found that neither the complement fixation nor the precipitation tests give satisfactory results. The agglutination test, however, offered a ready means for grouping as the results obtained with this test were in accord with those obtained by toxin-antitoxin tests. The agglutination test has permitted us to classify also such strains of Bacillus botulinus which have lost their toxicity under the conditions of test tube cultivation. As a control in all the above experiments we included a strain of Bacillus sporogenes and found that contrary to the statement in the literature all but one of the strains obtained by us from different laboratories in this country are free from Bacillus sporogenes contamination as judged by the above serologic tests.

12 (1759)

The antiscorbutic potency of strawberries.

By Clarence A. SMIth, OLAF BERGEIM, and PHILIP B. HAWK.

[From the Laboratory of Physiological Chemistry of Jefferson Medical College, Philadelphia, Pa.]

Several guinea pigs were fed a diet of oats, milk, and hay until they were decidedly scorbutic. They were then given expressed strawberry juice, either fresh juice or juice previously boiled for five minutes. The symptoms of scurvy were overcome within seven days by the administration of ten c.c. per day of either boiled or unboiled juice. Strawberries, therefore, appear to be relatively rich in water-soluble $\mathrm{C}$, and their content of this vitamine is not seriously decreased by five minutes boiling.

\title{
$13(1760)$
}

A modified anaphylactic reaction induced by $X$-rays.

By R. G. HUSSEY (by invitation).

[From the Rockefeller Institute for Medical Research, New York City.]

The following observations are of interest in connection with a theoretical consideration of the mechanism of classical serum anaphylaxis. 
We have found it possible, as has v. Heinrich also, to modify the manifestations of anaphylaxis in guinea pigs by exposing them to $\mathrm{X}$-rays. Guinea pigs weighing about 250 grams were given an intraperitoneal inoculation of 0.1 c.c. of horse serum (I c.c. of a I - Io dilution) for sensitization. Half of the number of sensitized animals were radiated immediately and then each day thereafter for Io days. The X-rays were delivered from a Coolidge tube governed by a spark gap of 3 inches with to milliamperes of current. The distance from the anode to the surface of the animal's body was 6 inches and the total time of exposure was Io minutes.

I4 days after sensitization 0.I c.c. or 0.oI c.c. of horse serum was inoculated into the jugular vein as an intoxicating dose. In the animals sensitized but not $\mathrm{X}$-rayed, typical anaphylactic manifestations and usually acute death followed the inoculation with either amount of antigen. The $\mathrm{X}$-rayed animals, on the other hand, showed either very slight or no objective anaphylactic manifestations. If, however, 4 weeks were allowed to elapse from the time of sensitization, then a similar amount of antigen inoculated intravenously, there was no difference in the behavior of $\mathrm{X}$-rayed animals as compared with the controls. Further, it was found that radiation at any time other than during the incubation period did not induce a modified reaction.

With these facts established, we directed our attention to a study of the anaphylactic state of isolated tissue. It may be said that many investigators describe the anaphylactic reaction of isolated smooth muscle as an index of the reaction of the animal body as a whole. Indeed, this phenomenon is regarded by them as the most important evidence which indicates that the locus of antigen-antibody union is intracellular.

Female guinea pigs of about 225 grams were sensitized by an intrapleural inoculation of 0.1 c.c. of horse serum and subsequently treated as described in the original experiment. At intervals of 14 and 30 days following sensitization, the horns of the uteri were removed and segments of these treated in accordance with the principles of the well-known Dale method for studying the physiological behavior of isolated tissues. For each tissue preparation, we employed a suspension bath of 250 c.c. of oxygenated Locke's 
solution kept at a constant temperature of $38^{\circ} \mathrm{C}$. When the muscle developed a satisfactory tone and rhythm, 0.2 c.c. or 0.5 c.c. of horse serum was added to the bath at a point which permitted uniform diffusion throughout the fluid before coming in contact with the tissue. The uteri of the sensitized and X-rayed animals reacted typically with maximal response just as did the uteri of the sensitized animals not $\mathrm{X}$-rayed. The tracings of the uteri removed at both intervals show no essential differences.

The information furnished by the data presented we believe to have a direct bearing on the controversial point regarding the locus of antigen-antibody union which results in anaphylactic shock. The results of our experiments indicate that the anaphylactic reaction of isolated smooth muscle is not an index of the reaction of the animal as a whole. Also it is indicated that other factors than the reaction of sensitized smooth muscle should be taken into account in the statement of a theory concerning the mechanism of anaphylactic shock.

We have now in process an investigation in which we are determining the relation between the existence of free antigen and the presence of precipitins in the circulation of animals X-rayed and not $\mathrm{X}$-rayed. The results of our experiments to date indicate that free antigen remains in the serum of $\mathrm{X}$-rayed animals for a much longer period than is found in animals not X-rayed. A full report of these results, together with studies on passive anaphylaxis, will be published later.

$$
\text { I4 (I76I) }
$$

\section{Contribution to study of diphtheria toxin.}

By P. J. MOLONEY and L. HANNA (by invitation).

[From the Reseurch Division, Connaught Antitoxin Laboratories, University of Toronto, Toronto, Canada.]

The results reported are those of experiments planned to throw further light on the mechanism of toxin production by B. diphtheria.

An extended series of test-tube experiments was carried out in which the Park 8 strain of the diphtheria bacillus was grown in broth and daily counts made of the number of viable organisms 